Mens

revue d'histoire intellectuelle de l'Amérique française

\title{
VII. L’époque contemporaine (1960-2003)
}

Volume 6, numéro supplément, 2005-2006

URI : https://id.erudit.org/iderudit/1024242ar

DOI : https://doi.org/10.7202/1024242ar

Aller au sommaire du numéro

Éditeur(s)

Centre de recherche en civilisation canadienne-française

ISSN

1492-8647 (imprimé)

1927-9299 (numérique)

Découvrir la revue

Citer ce document

(2005). VII. L'époque contemporaine (1960-2003). Mens, 6, 82-96.

https://doi.org/10.7202/1024242ar d'utilisation que vous pouvez consulter en ligne.

https://apropos.erudit.org/fr/usagers/politique-dutilisation/ 
THÉRIAULT, Janine, D'un catholicisme à l'autre : trois ordres catholiques au Québec et leur revues face à l'Aggiomamento et à la Rérolution tranquille, 1958-1970, Ph.D. (Histoire), Université de Montréal, 2003, 420 p.

TREMIBLAY, Marthe-Francine, Culture colonisée et ethnocentrisme : Québec (1950-1975), M.A. (Histoire), Université du Québec à Montréal, 1983, $333 \mathrm{p}$.

TREMCBLAY, Yolaine, $D u$ Refus Global à la responsabilité entière : parcours analytique de l'essai québécois depuis 1948, Sainte-Foy, Éditions Le Griffon d'argile, 2000, $170 \mathrm{p}$.

TRÉPANIER, Pierre, "Les tribulations d'André Dagenais ", Les Cabiers des Dix, 56 (2002), p. 235-295.

TRÉPANIER, Pierre, «Le philosophe André Dagenais devant la critique », Les Cahiers des Dix, 57 (2003), p. 205-262.

TURCOT'TE, Sylva, Les commissaires d'écoles catholiques de l'Estrie (1950-1990), M.A. (Histoire), Université de Sherbrooke, 1998, 173 p.

VIENS, Nathalie, «L'Humanisme intégral comme doctrine censoriale : la revue Lectures des Éditions Fides (1946-1951)", Voix et images, 23, 2 (hiver 1998), p. 281-296.

WARREN, Jean-Philippe, Un supplément d'àme : les intentions primordiales de Fernand Dumont, 1947-1970, Sainte-Foy, Les Presses de l'Université Laval, 1998, 176 p.

\section{L'époque contemporaine (1960-2003)}

ARSENAULT, Mathieu, Histoire de l'Association pour les droits des gai(e)s du Québec (1976-1986), M.A. (Histoire), Université du Québec à Montréal, 2000, $161 \mathrm{p}$.

AUGUSTIN, Jean-Pierre, "L'attraction croissante du Québec en France : le mélange d'une vision traditionnelle à celle d'une culture ouverte au monde ", Études canadiennes/Canadian Studies, 49 (2000), p. 109-129. 
BAILLARGEON, Constantin, Pierre I allières i'u par son professeur de philosophie, M Iontréal, Médiaspaul, 2002,127 [12] p.

BAILLARGEON, Gaëtan, " "De la cathédrale au chantier !" Les intellectuels québécois et Vatican II (1963-1966) ", dans Gilles ROUTHIER (dir.), I'atican II au Canada : enracinement et réception, Saint-Laurent, Fides, coll. " Héritage et projet », 2001, p. 273-292.

BASTIEN, Frédéric, Relations particulières : la France face au Québec après de Gaulle, Montréal, Boréal, 1999, 423 p.

BEAUCHEMIIN, Jacques, "La question nationale québécoise : les nouveaux paramètres de l'analyse ", Recherches sociographiques, 39, 2-3 (mai-décembre 1998), p. 249-269.

BEAUCHEMIIN, Jacques, « Dumont : historien de l'ambiguité », Recherches sociographiques, 42, 2 (mai-août 2001), p. 219-238.

BEAUCHEMIN, Jacques, L'bistoire en trop : la mauraise ionscience des sourerainistes québécois, Montréal, VLB, 2002, 214 p.

BEAUDREAU, Sylvie, "Déconstruire le rêve de nation. Lionel Groulx et la Révolution tranquille ", Rerue d'histoire de l'Amérique française, 56, 1 (été 2002), p. 29-61.

BÉDARD, Éric, Chronique d'une insurrection appréhendée. La irise d'Octobre et le milieu universitaire, Sillery, Septentrion, 1998, 204 p. BÉLAND, Caroline, Les Éditions Mille Roches : une mission d'éditeur régional, M.A. (Lettres), Université de Sherbrooke, 2000, 269 p. BÉLANGER, Yves, Robert COMEAU et Céline MIÉTIVIER (dirs.), La Rérolution tranquille. 40 ans plus tard: un bilan, Montréal, VLB éditeur, 2000, 316 p.

BELLEROSE, Charles et Jacques BEAUCHEMIIN, "Communauté nationale et définition du sujet politique : analyse de deux grandes consultations populaires au Québec (1967-1995) ", Québec Studies, 28 (1999-2000), p. 27-55.

BERGERON, Mario, Société québécoise, salles de cinéma au Québec et à Trois-Rivières : quatre aspects, M.A. (Études québécoises), Université du Québec à Trois-Rivières, 1999, 275 p. 
BERNIER, Luc, De Paris à Washington : la politique internationale du Québec, Sainte-Foy, Les Presses de l’Université du Québec, 1996, $173 \mathrm{p}$.

BOCK, Michel, Comment un peuple oublie son nom : la irise identitaire franco-ontarienne et la presse française de Sudbury (1960-1975), Sudbury, Éditions Prise de parole et Institut franco-ontarien, 2001, 119 p.

BOILY, Caroline, La place et la conception de la discipline historique dans l'enseignement seiondaire québécois : analyse du débat actuel, M.A. (Sociologie), Université du Québec à Montréal, 1998, 164 p.

BOISMENU, Gérard et Jane JENSON, «La dislocation du régime de citoyenneté canadien et l'évolution du régime fédératif », Cahiers d'histoire, 17, 1-2 (printemps-automne 1997), p. 126146.

BOSHER, J.F., The Gaullist Attack on Canada, 1967-1997, Kingston et Montréal, McGill-Queen's University Press, 1998, 304 p.

BOUCHARD, Gérard et Michel LACOMIBE, Dialogue sur les pays neufs, Montréal, Boréal, 1999, 224 p.

BOUCHARD, Gérard, "Sur le modèle de la nation québécoise et la conception de la nation chez Fernand Dumont ", Bulletin d'histoire politique, 9, 2 (hiver 2001), p. 144-159.

BOUCHARD, Gérard, "L'américanité : un débat mal engagé ", Argument, 4, 2 (printemps-été 2002), p. 159-180.

BOUCHER, Marc, "L'évolution de l'image du Québec aux États-Unis », Politique et sociétés, 18, 1 (1999), 151-164.

CALDWELL, Gary et Julien HARVEY, "Le prérequis à l'intégration des immigrants : une culture publique commune au Québec ", L'Action nationale, 84, 6 (juin 1994), p. 786-794.

CAREL, Ivan, «Un mouvement de droite nationale contemporaine : Jeune Nation ", Bulletin d'bistoire politique, 11, 1 (automne 2002), p. 115-126.

CAREL, Ivan, "La Révolution tranquille en paradigmes ", Bulletin d'histoire politique, 12, 1, (automne 2003) , p. 201-210. 
CHASSAY, Jean-François (dir.), Anthologie de l'essai au Québei depuis la Rérolution tranquille, Montréal, Boréal, 2003, 271 p.

CHÉNIER, Jean-Félix, La pensée de Charles Taylor sur les deux libéralismes au Canada, M.A. (Science politique), Université Laval, 2000, 155 p.

CHÉNIER, Jean-Félix, "Charles Taylor et l'abandon de la survivance », Globe. Rerue internationale d'études québécoises, 6, 2 (2002), p. $147-174$.

CHEVRIER, Marc, «Le chantier abandonné de 1964 : les onze propositions de Daniel Johnson (père) sur l'éducation ", Les Cahiers d'bistoire du Québec au XXe siècle, 7 (printemps 1997), p. 164178.

CHURCHILL, Stacey, «Bilan de trente années de la promotion des langues officielles au Canada : vers un État-nation postmoderne ", Études canadiennes/Canadian Studies, 45 (1998), p. 49-64

CLOU'TIER, Caroline, Les réactions de la communauté juive du Québec face à la législation linguistique et au projet sonıerainiste du Parti québéiois entre 1974 et 1981, M.A. (Histoire), Université du Québec à Montréal, 1999, 126 p.

CLOUTIER, Caroline, "L'unilinguisme français et le projet souverainiste du Parti québécois vu par la communauté juive du Québec (1974-1981) ", Bulletin d'histoire politique, 8, 1 (automne 1999), p. 123-136.

COHEN, Andrew et J.L. GRANATSTEIN (dirs.), Trudeau's Shadow: The Life and Legacy of Pierre Elliott Trudeau, Toronto, Random House, 1998, 320 p.

COHEN-ALMLAGOR, Raphaël, "The Terrorists' Best Ally: The Quebec Media Coverage of the FLQ Crisis in October 1970 ", Canadian Journal of Communication, 25, 2 (2000), p. 251-285.

COMEAU, Robert et Bernard DIONNE (dirs.), $\dot{A}$ propos de l'Histoire nationale, Sillery, Septentrion, 1998, 160 p. 
CONLEY, Richard S., "Sovereignty or the Status Quo ? The 1995 Pre-referendum Debate in Quebec ", Joumal of Commonwealth e Comparative Politics, 35, 1 (mars 1997), p. 67-92.

CONWAY, Kyle, «Les interprétations multiples de société distincte lors de l'accord de Meech : une lecture du Toronto Star et de La Presse de Montréal ", Journal of Canadian Studies/Rerue d'études canadiennes, 37, 4 (hiver 2002-2003), p. 112-130.

CUCCIOLETTA, Donald et Albert DESBIENS, "L'Américanité, The Dual Nature of the Québécois Identity ", Québec Studies, 29, (été 2000), p. 3-14.

CUPIDO, Robert, "Appropriating the Past: Pageants, Politics, and the Diamond Jubilee of Confederation ", Journal of the Canadian Historical Association/Retue de la Société historique du Canada, 9, (1998), p. 155-186.

DASSYLVA, Martial, "L'Église catholique du Québec et "l'épreuve" de la Révolution tranquille. Deuxième partie : la création du ministère de l'Éducation ", Bulletin d'histoire politique, 10, 3 (été 2002), p. 129-139.

DEGAGNÉ, Sébastien, Le mouvement marxiste-léniniste En Lutte! et la question nationale québécoise au Canada (1972-1982), M.A. (Histoire), Université du Québec à Montréal, 1998, 121 p.

DELEUZE, Magali, "Une définition historique de l'intellectuel québécois ? ", Cahiers d'histoire, 16, 1 (printemps 1996), p. 25-33.

DEMERS, Frédéric, "Sur l'historiographie de la télévision au Québec et le pesant récit de la Révolution tranquille ", Mens. Rei'ue d'histoire intellectuelle de l'Amérique française, 3, 2 (printemps 2003), p. 234-267.

DENIS, Claude, «La patrie et son nom : essai sur ce que veut dire le "Canada français" ", Francophonies d'Amérique, 6, (1996), p. 185-198.

DION, Léon, La réiolution déroutée (1960-1976), Montréal, Boréal, 1998, $324 \mathrm{p}$. 
DORÉ, Martin, "Analyse de la collection "Constantes" des Éditions Hurtubise $\mathrm{HNIH}$ », L'Action nationale, 91, 10 (décembre 2001), p. 79-95.

DOUCET, Sophie et Karine HÉBERT, «L'histoire du féminisme au Canada et au Québec : bibliographie sélective ", Mens. Reı'ue d'bistoire intellectuelle de l'Amérique française, 2, 1 (automne 2001), p. 125-144.

DUPONT, Louis, «L'américanité québécoise : portée politique d'un courant d'interprétation ", dans Donald Cuccioletta (dir.), L'Américanité et les Amériques, Sainte-Foy, Éditions de l'IQRC, 2001, p. 47-63.

DUPONT, Louis, «L'américanité québécoise : portée politique d'un courant d'interprétation », dans Donald Cuccioletta (dir.), L'Américanité et les Amériques, Sainte-Foy, Éditions de l'IQRC, 2001, p. 47-64.

FAIVRE-DUBOZ, Brigitte, "Au croisement de la culture et du politique. Pierre Elliott Trudeau et Hubert Aquin face à l'Étatnation ", Globe. Reine internationale d'études québécoises, 4, 1 (2001), p. 11-27.

FALARDEAU, Guy, Le poids des générations et le changement social au Québec: une approche sociodémographique du phénomène des générations, Ph.D. (Sciences politiques), Université de Montréal, 1996, 234 p.

FERRETTI, Lucia, "La Révolution tranquille », L'Action nationale, 89, 10 (décembre 1999), p. 59-91.

FITCH, Lori, Nenspaper Coverage of the FLQ Crisis by La Presse and the Toronto Daily Star: a Study of Opinions on Canadian Democracy, M.A. (Études québécoises), Université du Québec à Trois-Rivières, 1997, $123 \mathrm{p}$.

FLQ, Manifeste d'octobre 1970, notes et postface de Christophe Horguelin, Montréal, Comeau \& Nadeau, 1998, 54 p.

FORTIER, Julie, Information télérisée et identité nationale : comparaison entre les chaines SRC, CBC et TT $A, M$.A. (Science politique), Université de Montréal, 1999, 120 p. 
FOURNIER, N Iarcel, "Fernand Dumont et la modernité », Recheribes sociographiques, 42, 2 (mai-août 2001), p.267-282.

FRATTA, Carla et Élisabeth NARDOUT-LAFARGE (dir.), Italies imaginaires du Québec, Saint-Laurent, Fides, coll. « Nouvelles études québécoises », 2003, 246 p.

GAGNÉ, Francine, Les conditions d'existence d'un périodique féministe d'actualité destiné au grand public : les legons à tirer de l'expérience du magazine La vie en rose, M.A. (Communication), Université du Québec à Montréal, 1997, 129 p.

GAGNÉ, Gilles, «Le vote en faveur de la souveraineté du Québec (1995-2000) ", Recheribes sociographiques, 41, 3 (automne 2000), p. 431-477.

GAGNÉ, Gilles et Simon LANGLOIS, Les raisons fortes. Nature et signification de l'appui à la souveraineté du Québec, MIontréal, Les Presses de l'Université de Mlontréal, 2002, 192 p.

GAGNON, Serge, "À propos de Ronald Rudin », Bulletin d'histoire politique, 7, 1 (automne 1998), p. 133-151.

GAGNON, Serge, Le passé composé : de Ouellet à Rudin, Montréal, VLB éditeur, 1999, $190 \mathrm{p}$.

GERVAIS, André, « D’où vient l'expression "révolution tranquille" ? ", Bulletin d'bistoire politique, 6, 2 (hiver 1998), p. 115-122.

GERVAIS, Gaétan, «L'Ontario français et les "États généraux du Canada français" (1966-1969) ", Cahiers Charleioix. Études francoontariennes, 3 (1997), p. 231-364.

GOULD, Jean, «Des bons pères aux experts », Société, 20-21 (été 1999), p. 111-188.

GÜNTZEL, Ralph P., «The Centrale de l'Enseignement du Québec and Quebec Separatist Nationalism, 1960-1980 », The Canadian Historical Rerien, 80, 1 (mars 1999), p. 61-82.

GÜNTZEL, Ralph P., «"Rapprocher les lieux du pouvoir" : The Quebec Labour Movement and Quebec Sovereigntism (19602000) ", Labour/Le Traiail, 46 (automne 2000), p. 369-395. 
GUILMLAIN, Sylvain, Le rôle de Georges-Émile Lapalme et d'André Patry dans la mise en place d'une politique des relations internationales $d u$ Québec de 1960 à 1968, M.A. (Histoire), Université du Québec à Montréal, 1999, $136 \mathrm{p}$.

JOMPHE, Carl, Pour une école démocratique : Gérard Filion et les réformes scolaires des années 1960, M.A. (Histoire), Université du Québec à Montréal, 1998, 119 p.

JOUBER'T, Lucie, «L'Église et ses émissaires : la cible privilégiée de l'ironie au féminin dans la littérature québécoise (1960-1980) ", Québec Studies, 25 (printemps 1998), p. 46-58.

KELLY, Stéphane (dir.), Les idées mènent le Québec : essais sur une sensibilité historique, Sainte-Foy, Les Presses de l'Université Laval, coll. «Prisme », 2002, 250 p.

LACHAPELLE, Guy et Gilbert GAGNÉ, «L'américanité du Québec ou le développement d'une identité nord-américaine ", Francophonies d'Amérique, 10 (2000), p. 87-99.

LOUDER, Dean et Cécyle TRÉPANIER, «Sur les routes de l'Amérique française : l'expérience des géographes lavallois ", Québec Studies, 33 (printemps-été 2002), p. 15-51

LAMONDE, Yvan et Marie-Pierre TURCOT, La littérature personnelle au Québec (1980-2000), M Iontréal, Bibliothèque nationale du Québec, 2000, 100 p.

LAMONDE, Yvan, «À propos de Critique de l'américanité. Mémoire et démocratie au Québec de Joseph-Yvon Thériault », Bulletin d'histoire politique, 11, 2 (hiver 2003), p. 177-183.

LANGLOIS, André, «Analyse de l'évolution démolinguistique de la population francophone hors-Québec, 1971-1996 », Reiherihes sociographiques, 41, 2 (2000), p. 211-238.

LAPIERRE-ADAMCYK, Évelyne et al., "Vivre en couple pour la première fois : la signification du choix de l'union libre au Québec et en Ontario ", Cahiers québécois de démographie, 28, 1-2 (printemps-automne1999), p. 199-228. 
LAPOINTE, Simon, "Parti pris et le nationalisme québécois », Cabiers d'histoire, 12, 1 (hiver 1992), p. 51-56.

LAROSE, Sylvain, La création de la délégation générale du Québec à Paris (1958-1964), M.A. (Histoire), Université du Québec à Montréal, 1999, $150 \mathrm{p}$.

LATENDRESSE, Claude, L'Ordre des Jésuites et la sécularisation au Québec: analyse des discours de la reine Relations (1958-1970), M.A. (Sociologie), Université de Montréal, 1986, 145 p.

LEBEL, Marc, " Quatre décennies de travaux : l'itinéraire bibliographique de Pierre Savard ", dans Jean-Pierre Wallot (dir.), Construitions identitaires et pratiques sociales, Ottawa, Les Presses de l'Université d'Ottawa, 2002, p. 409-432.

LEROUX, Manon, Le discours des aiteurs de la crise d'octobre 1970 dans la presse francophone de Montréal (1971-2000), M.A. (Histoire), Université du Québec à Montréal, 2001, vi-168 p.

LEROUX, Manon, «D'une commémoration à l'autre » [sur la Crise d'Octobre 1970], Bulletin d'bistoire politique, 11, 1 (automne 2002), p. 82-92.

LEROUX, Manon, Les silenies d'Octobre. Le discours des aiteurs de la irise de 1970, Montréal, VLB, coll. «Études québécoises ", 2002, $169 \mathrm{p}$.

LÉTOURNEAU, Jocelyn, "Nous autres Québécois" - La voix des manuels d'histoire ", Internationale Schulbuchforschung/International Textbook Research, 18, 3 (janvier 1996), p. 269-287.

LÉTOURNEAU, Jocelyn, "Le lieu (dit) de la nation : essai d'argumentation à partir d'exemples puisés au cas québécois ", Canadian Journal of Political Science/Reine canadienne de science politique, 30, 1 (mars 1997), p. 55-87.

LINTEAU, Paul-André, «Un débat historiographique : l'entrée du Québec dans la modernité et la signification de la Révolution tranquille ", Francophonia. Studi e ricerche sulle letterature di lingua francese, 19, 37 (automne 1999), p. 73-87. 
LINTEAU, Paul-André, « Un débat historiographique : l'entrée du Québec dans la modernité et la signification de la Révolution tranquille ", dans Yves Bélanger et al. (dirs.), La Rérolution tranquille, 40 ans plus tard: un bilan, Montréal, VLB Éditeur, 2000, p. 21-41.

LUNEAU, Marie-Pier, "Le nom supposé comme outil de transgression "d'un illuminé" ou "Frère Untel". De l'usage de la fausse signature chez les prêtres au Québec (1809-1979) », Études d'histoire religieuse/ Historical Studies. Canadian Catholic Historical Association, 70 (2004), p. 39-55.

LUSSIER, André, Le nationalisme québécois sur le diran, Montréal, Fides, 2002, 202 p.

MACHABÉE, Daniel, La Commission royale d'enquête sur le bilinguisme et le biculturalisme ou la tentative de reconnaissance de la dualité canadienne (1963-1971), M.A. (Histoire), Université du Québec à Montréal, 1999, $135 \mathrm{p}$.

MACLURE, Jocelyn, Récits identitaires : le Québec à l'épreure du pluralisme, Montréal, Québec Amérique, 2000, 219 p.

MARCIL, Olivier, Libéralisme, nationalisme et catholicisme dans la pensée politique de Claude Ryan au Devoir (1962-1978), M.A. (Histoire), Université de Montréal, 2001, v-227 p.

MARCIL, Olivier, « La question linguistique dans la pensée de Claude Ryan au Deioir (1962-1978) : la difficile conciliation de principes nationalistes et libéraux ", Mens. Rei'ue d'histoire intellectuelle de l'Amérique française, 2, 2 (printemps 2002), p. 193-232.

MARCIL, Olivier, La raison et l'équilibre : libéralisme, nationalisme et catholicisme dans la pensée de Claude Ryan au Devoir (1962-1978), Montréal, Éditions Varia, coll. «Histoire et Société », 2002, 292 p.

MLARTEL, Marcel (dir.), avec la collaboration de Robert CHOQUETTE, Les États généraux du Canada français, trente ans après. Actes du colloque tenu à l'Unicersité d'Ottania en notembre 1997, Ottawa, Centre de recherche en civilisation canadienne-française de l'Université d'Ottawa, 1998, 422 p. 
NAARTEL, N Iarcel, «Les politiques gouvernementales fédérale et québécoise à l'égard des minorités francophones du Canada (1960-1980)» Francophonies d'Amérique, 9 (1999), p. 199-208.

MATHIEU, Geneviève, Qui est Québécois? Synthèse du débat sur la redéfinition de la nation, $\mathrm{N}$ Iontréal, VLB, coll. "Études québécoises", 2001, 140 p.

M IcEVOY, Frederick J., "The Establishment of Diplomatic Relations between Canada and the Vatican, 1969 ", Études d'histoire religieuse/Historical Studies. Canadian Catholic Historical Association, 68 (2002), p. 66-84.

MEUNIER, E.-A Iartin, « Intellectuel-militant catholique et théologie de l'engagement : la consécration d'un prophète en SaintJérôme ", Societéé, 20-21 (été 1999), p. 255-311.

MIEUNIER, E.-M artin et Jean-Philippe WARREN, Sortir de la "Grande Noireur": L'horizon personnaliste de la Rérolution tranquille, Sillery, Septentrion, coll. « Cahiers du Septentrion », 2002, 216 p.

NEUNIER, E.-N Lartin, "Jacques Grand'maison et la Révolution tranquille ", Mens. Reinu d'histoire intellectuelle de l'Amérique française, 3, 2 (printemps 2003), p. 149-191.

NIILLS, Sean, "Québeicoises deboutte ! Le Front de libération des femmes du Québec, le Centre des femmes et le nationalisme", Mens. Retue d'bistoire intellectuelle de l'Amérique française, 4, 2 (printemps 2004), p. 183-210.

MIONTPETIT, Éric et Christian ROUILLARD, « La Révolution tranquille et le réformisme institutionnel. Pour un dépassement des discours réactionnaires sur l'étatisme québécois ", Globe. Rène internationale d'études québéioises, 4, 1 (2001), p. 119-139.

MOREAU, Daniel, Le rapport Parent et l'enseignement de l'histoire nationale au Québec entre 1960-1970: autopsie d'une réforme alortée, M.A. (Histoire), Université du Québec à Nontréal, 2003, vi-144 p.

NOËL, Nathieu, Le mourement Québec Français de 1971 à 1996, M.A. (Sciences politiques), Université du Québec à Montréal, 1998, 154 p. 
PAGE, Carole, Décolonisation et question nationale québécoise, M.A. (Science politique), Université du Québec à M Iontréal, 1978, 154 p. PAQUÊT, Martin, « Les États généraux du Canada français et l'immigration, novembre 1967 », Bulletin d'histoire politique, 10, 2 (hiver 2002), p. 123-134.

PARADIS, Jean-Marc, "Les politiciens et le sport en Mauricie", Bulletin d'bistoire politique, 11, 2 (hiver 2003), p. 45-52.

PARENT, Sébastien, L'historiographie moderniste québécoise (19822002) : une production récisionniste ?, M.A. (Histoire), Université du Québec à Montréal, 2003, v-194 p.

PINSONNEAULT, Olivier, L'utilisation d'Internet par les partis politiques au Canada : les cas du Parti libéral du Canada, de l'Alliance canadienne et du Parti maraiste-léniniste du Canada, M.A. (Communication), Université du Québec à Montréal, 2003, ix-201 p.

PLEAU, Jean-Christian, La Rérolution québécoise: Hubert Aquin et Gaston Miron au tournant des années soivante, [Saint-Laurent], Fides, coll. " Nouvelles études québécoises », 2002, 270 p.

POTWOROWSKI, Christophe, "La présence de M.-D. Chenu au Canada ", dans Gilles Routhier (dir.), I atican II au Canada : enracinement et réception, Saint-Laurent, Fides, coll. « Héritage et projet ", 2001, p. 41-52.

POULIN, Philippe, Les relations France-Québec au cours du second mandat du gouternement Léiesque (1980-1985), M.A. (Histoire), Université de Montréal, 1997, vi-290 p.

PRIMEAU, André [Francis], "André d'Allemagne et le projet indépendantiste québécois ", Bulletin d'histoire politique, 12, 1 (automne 2003), p. 170-192.

PRONOVOST, Gilles, «Les transformations des usages du temps (1986-1992) ", Recherihes sociographiques, 39, 1 (janvier-avril 1998), p. 121-148. 
PROVOST, Francis, Étude sur les dissensions entre la Droite et la Gauche au sein du Rassemblement pour l'indépendance nationale entre 1966 et 1968, M.A. (Histoire), Université du Québec à Montréal, 2003, iv-109 $\mathrm{p}$.

RAMIOS, Howard et Kevin GOSINE, " "The Rocket":

Newspaper Coverage of the Death of a Québec Cultural Icon, A Canadian Hockey Player ", Journal of Canadian Studies/Retue d'études canadiennes, 36, 4 (hiver 2001-2002), p. 9-31.

ROBERGE, Daniel, Le traitement de la question nationale québécoise cheq les historiens et les sociologues (1960-1990), M.A. (Histoire), Université du Québec à M Iontréal, 1999, 199 p.

ROUTHIER, Gilles, "Un toumant de Vatican II », Études d'bistoire religieuse / Historical Studies. The Canadian Catholic Historical Association, 64 (1998), p. 71-79.

ROUTHIER, Gilles, «Entre Révolution tranquille et aggiomamento conciliaire : évolution dans le gouvernement du diocèse de Québec au cours des années 1960 ", Études d'histoire religieuse/ Historical Studies. The Canadian Catholic Historical Association, 67 (2001), p. 47-56.

ROUTHIER, Gilles (dir.), I atican II au Canada : enrainement et réception, Saint-Laurent, Fides, coll. « Héritage et projet », 2001, 543 p.

ROUTHIER, Gilles, «Assurer la couverture du concile Vatican II au Canada : Les initiatives de l'épiscopat canadien ", Études d'histoire religieuse/Historical Studies. The Canadian Catholic Historical Association, 68 (2002), p. 57-71.

ROUTHIER, Gilles (dir.), Élêques du Québec, 1962-1965 : entre Rérolution tranquille et aggiornamento conciliaire, Sainte-Foy, Centre interuniversitaire d'études québécoises, coll. « Cheminements ", 2002,65 p. 
ROY, Christian, "Le Québec et le Canada au regard du fédéralisme européen et du nationalisme canadien : un entretien avec Jacques-Yvan Morin ", Argument, 4, 2 (printemps-été 2002), p. 115-130.

RYAN, Claude, «L'Église catholique et l'évolution politique du Québec de 1960 à 1980 », Sessions d'études - Société canadienne d'bistoire de l'Église catholique, 50 (1983), p. 381-402.

SCHMITZ, Nancy, "La langue du trèfle et la résistance francophone : la Saint-Patrick à Québec à l'aube du XXIe siècle ", dans Jean-Pierre Pichette (dir.), Entre Beauce et Aiadie : facettes d'un pariours ethnologique. Études offertes au professeur Jean-Claude Dupont, Sainte-Foy, Les Presses de l'Université Laval, 2001, p. 129-135.

SELJAK, David, "Catholicism's "Quiet Revolution”: Maintenant and the New Public Catholicism in Quebec after 1960 ", dans Marguerite Van Die (dir.), Religion and Public Life in Canada: Historical and Comparative Perspectives, 'Toronto, University of Toronto Press, 2001, p. 257-274.

SERRÉ, Pierre, Deux poids, deux mesures : l'impact du iote des nonfrancophones au Québec, Montréal, VLB éditeur, 2002, 262 p.

THÉRIAULT, Joseph-Yvon, Critique de l'américanité : mémoire et démocratie au Québec, Montréal, Éditions Québec Amérique, 2002, 373 p.

THOMAS, Karl-Xavier, "Attendre? Oui. Reculer : Jamais! Les étudiants de l'Université de Montréal et le combat pour la gratuité scolaire sous le gouvernement Lesage ", Bulletin du RCHTQ, 26, 1 (printemps 2000), p. 3-18.

TOONDER, Jeanette den, «Pluriculturalisme au Québec : la voix des auteurs allophones ", Journal of Canadian Studies/Rerue d'études canadiennes, 35, 3 (2000), p. 105-119.

TRÉPANIER, Pierre, «Faire de l'histoire à la manière de Ronald Rudin ", Bulletin d'histoire politique, 7, 1 (automne 1998), p. 106-118. 
TRÉPANIER, Pierre, «L'histoire intellectuelle selon Jean de Viguerie ", Mens. Retue d'histoire intellectuelle de l'Amérique française, 2, 1 (automne 2001), p. 5-15.

VALLIÈRES, Pierre, Paroles d'un nègre blanc, anthologie préparée par Jacques Jourdain et Mélanie Mailhot, Montréal, VLB, coll. "Partis pris actuels ", 2002, $284 \mathrm{p}$.

VENNE, M lichel (dir.), Penser la nation québécoise, M Iontréal, Québec Amérique, 2000, $308 \mathrm{p}$.

VINCENT, Josée, Les tribulations du litre québeicois en France (1959. 1985), Québec, Nuit blanche éditeur, 1997, 234 p.

VINCENT, Sophie, L'apprentissage de la liberté. Mutations de la censure au Québec, de l'abolition de l'Index aux lendemains de la crise d'Octobre (1966-1971), M.A. (Lettres et communications), Université de Sherbrooke, 2002, $186 \mathrm{p}$.

WALLOT, Jean-Pierre (dir.), Le débat qui n'a pas eu lieu : la Commission Pepin-Robarts, quelque ringt ans après, Ottawa, Presses de l'Université d'Ottawa, coll. «Amérique française », 9 (2002), 148 p.

WARREN, Jean-Philippe, «Gérard Pelletier et Cité libre : la mystique personnaliste de la Révolution tranquille ", Société, 20-21 (été 1999), p. 313-346.

WARREN, Jean-Philippe et E-M Iartin NEUNIER, «L'horizon "personnaliste" de la Révolution tranquille », Société, 20-21 (été 1999), p. 347-448.

WARREN, Jean-Philippe, «L'inquiétude des révolutionnaires tranquilles : bref essai d'analyse d'un sentiment ", Argument, 4, 2 (printemps-été 2002), p. 75-92.

WARREN, Jean-Philippe, «La découverte de la "question sociale" : sociologie et mouvements d'action jeunesse canadiensfrançais ", Rel'ue d'histoire de l'Amérique franģaise, 55, 4 (printemps 2002), p. 539-572. 